INPLASY

PROTOCOL

To cite: Wu et al. Traditional Chinese exercises in adults with chronic kidney disease: A protocol for systematic review and meta-analysis. Inplasy protocol 2020100016. doi: 10.37766/inplasy2020.10.0016

Received: 06 October 2020

Published: 06 October 2020

Corresponding author: Mingquan Li

limingquan128@sina.com

Author Affiliation:

Department of Nephrology,

Hospital of Chengdu

University of Traditional

Chinese Medicine, Chengdu,

Sichuan, P.R. China

Support: None.

Review Stage at time of this submission: Preliminary

searches.

Conflicts of interest:

None.

\section{Traditional Chinese exercises in adults with chronic kidney disease: A protocol for systematic review and meta-analysis}

\author{
Wu, LH1; Lu, HM²; Qu, B33 Liu, Y4; Wu, L5; Li, MQ6.
}

Review question / Objective: To systematically review literature about the effects of traditional Chinese exercises (TCEs, i.e., Tai Chi, Baduanjin, Qigong) in adult patients with chronic kidney disease(CKD).

Condition being studied: Chronic kidney disease (CKD) is increasingly recognized as a global public health problem. Baduanjin and tai chi, alternative forms of exercise based on traditional Chinese medicine, are reported to be beneficial to patients with CKD. This systematic review analyzed the evidence and made recommendations for clinical applications and future research.

INPLASY registration number: This protocol was registered with the International Platform of Registered Systematic Review and Meta-Analysis Protocols (INPLASY) on 06 October 2020 and was last updated on 06 October 2020 (registration number INPLASY2020100016).

\section{INTRODUCTION}

Review question / Objective: To systematically review literature about the effects of traditional Chinese exercises (TCEs, i.e., Tai Chi, Baduanjin, Qigong) in adult patients with chronic kidney disease(CKD).

Condition being studied: Chronic kidney disease (CKD) is increasingly recognized as a global public health problem. Baduanjin and tai chi, alternative forms of exercise 
based on traditional Chinese medicine, are reported to be beneficial to patients with CKD. This systematic review analyzed the evidence and made recommendations for clinical applications and future research.

\section{METHODS}

Search strategy: The literature search will be conducted by two researchers (Lihua Wu \& Yu Liu) independently in the form of "back-to-back", discrepancies regarding included studies will be discussed and settled by a third reviewer. We will use the combination of medical subject headings(MeSH) and text words to complete the literature search strategies, mainly include: 1) "Qigong"(MeSH), "Qi Gong", "Ch'i Kung", "Chi gong", "Chi gung", 2) "Tai Ji"(MeSH), " Tai Ji Quan", "Tai chi", "Tai Chi Chuan", 3) "traditional Chinese exercise ", "Ba duanjin", "Ba duanjin qigong ", "Eight Fragments ", "Yijinjing " or "Wuqinxi "; 4 ) " Renal Insufficiency, Chronic"(MeSH) , "Kidney Failure, Chronic " (MeSH), "Kidney Transplantation" (MeSH), "hemodialysis ", "haemodialysis ", "dialysis ", "chronic renal disease " , "chronic kidney disease ", "chronic renal failure ", "chronic kidney insufficiency ","kidney transplant*","renal transplant* ","kidney graft* ","renal graft* ","CKD or CKF or CRD or CRF or ESKD or ESRD or ESKF or ESRF ". Besides, we will scan the reference lists of included original or relevant reviews to identify additional relevant articles.

Participant or population: All adults (ËH 18 years) with any stage CKD or who have received a kidney transplant were included. Studies investigating the effects of regular physical exercise training in adults with acute kidney injury (AKI) and studies in children were excluded.

Intervention: At least one type of TCE (i.e., Tai Ji or Wuqinxi, Baduanjin, qigong, or Yijinjing) was used to treat CKD. TCE plus another intervention versus the same intervention alone will be also enrolled. The exercise program should include: intensity, frequency, and duration (ËH 2 months).
Comparator: The control group should adopt one of the following treatment methods: a placebo, no treatment, usual care, or guideline-recommended conventional treatment. If the control group contains other non-conventional therapies, such as herbal medicine, acupuncture, moxibustion, massage, yoga, will be excluded.

Study designs to be included: Randomized controlled trial (RCT) and quasi-RCT including combination therapy and monotherapy of TCE will be included.

Eligibility criteria: 1.Types of study: RCT and quasi-RCT including combination therapy and monotherapy of TCE will be included. 2.Participants: all adults (ËH 18 years) with any stage CKD or who have received a kidney transplant were included. Studies investigating the effects of regular physical exercise training in adults with acute kidney injury (AKI) and studies in children were excluded.3. Interventions: At least one type of TCE (i.e., Tai Ji or Wuqinxi, Baduanjin, qigong, or Yijinjing) was used to treat CKD. TCE plus another intervention versus the same intervention alone will be also enrolled. The exercise program should include intensity, frequency, and duration (ËH 2 months).4. Comparator: the control group should adopt one of the following treatment methods: a placebo, no treatment, usual care, or guidelinerecommended conventional treatment. If the control group contains other nonconventional therapies, such as herbal medicine, acupuncture, moxibustion, massage, yoga, will be excluded.

Information sources: We will comprehensively search the following 9 databases: MEDLINE, EMBASE, Cochrane Central Register of Controlled Trials(CENTRAL), The Cumulative Index to Nursing \& Allied Health Literature (CINAHL), Web of Science, Chinese National Knowledge Infrastructure (CNKI), WANFANE Database, Chinese Scientific and Technological Periodical Database (VIP) and Chinese Biomedical Database (СВM). Ambiguous literature will be manually searched to avoid missing eligible 
trials. Ongoing registered clinical trials will be searched on the websites of the Chinese clinical trial registry (http:// www.chictr.org.cn/) and international clinical trial registry (http:// clinicaltrials.gov/). Additional trials will be searched by reviewing the reference lists of the retrieved articles, conference proceedings, and gray literature. We will contact the original investigators for more complete details of the study to solve questions about eligibility if necessary.

Main outcome(s): The primary outcomes include Physical fitness; Physical functioning and activity; Renal function; Cardiovascular dimensions: blood pressure (diastolic and systolic); maximum heart rate; resting heart rate; Nutritional measures; Systemic inflammation; Depression; Health-related quality of life(HRQoL).

Additional outcome(s): The secondary outcomes include: Muscle morphology and morphometrics; Glucose metabolism; Blood lipids; Dropout rates; Compliance; Adverse events (exercise-induced injuries); Mortality.

Quality assessment / Risk of bias analysis: The quality of the studies will be assessed by the Newcastle-Ottawa Quality Assessment Scale. For unclear items in the study, contact the corresponding author for details. The quality assessment will be conducted independently by two researchers (Lihua Wu \& Ling Wu). Any discrepancies will be resolved by/through discussion with a third reviewer (Mingquan Li).

Strategy of data synthesis: Review Manager 5.4 software, provided by the Cochrane Collaboration (www.cochrane.org), will be used for data analysis. Binary outcomes will be summarized using odds ratios (ORs) or relative risks (RRs) with a $95 \%$ confidence interval $(\mathrm{Cl})$ for relative effect. Continuous outcomes will be presented as a standardized mean difference (SMD) with $95 \% \mathrm{Cl}$ between groups. Statistical heterogeneity between trials will be analyzed by $Q$ statistic and 12 test. When the heterogeneity test indicated the existence of heterogeneity between studies (P Ëм.01, I2Ëн50\%), the random-effect model was used for combined analysis, or subgroup analysis or sensitivity analysis was conducted according to the heterogeneity source. When the heterogeneity test indicated that there was no heterogeneity between the groups $(P$ $>.01,12<50 \%$ ), the fixed-effect model was used for combined analysis. If quantitative synthesis is not appropriate, qualitative analysis will be carried out. Quality of evidence and strength of recommendations will be assessed by the Grading of Recommendations, Assessment, Development, and Evaluations (GRADE) method.

Subgroup analysis: Subgroup analysis will be further stratified by the type of intervention (i.e. Qi gong, Tai chi, Ba duanjin), treatment duration, or cointerventions.

Sensibility analysis: Sensitivity analysis will be performed by removing the inclusion of studies one by one, for evaluating whether the results of the meta-analysis is reliable and finding the potential sources of heterogeneity. The funnel plot analyses would be performed to determine potential publication bias if every comparison group included more than 10 studies.

Language: No language restrictions.

Country(ies) involved: China.

Other relevant information: None.

Keywords: chronic kidney disease, traditional Chinese exercise, systematic review.

Dissemination plans: The results will be published in a scientific journal after peerreview and disseminated electronically or in print.

Contributions of each author:

Author 1 - JC Lihua Wu - drafted the manuscript. 
Author 2 - JC Hongmei Lu - provided statistical expertise.

Author 3 - Ling Wu - contributed to the risk of bias assessment strategy.

Author 4 - Bo Qu - read, provided feedback and approved the final manuscript.

Author 5 - Yu Liu - contributed to the development of the selection criteria.

Author 6 - JC Mingquan Li - designed the study protocol. 